\title{
Time-Series prediction for the epidemic trends of COVID-19 using Conditional Generative adversarial Networks Regression on country-wise case studies
}

\section{Arnabi Bej}

Jadavpur University Faculty of Engineering and Technology

Ujjwal Maulik

Jadavpur University Faculty of Engineering and Technology

Anasua Sarkar ( $\square$ ashru2006@hotmail.com )

Jadavpur University https://orcid.org/0000-0001-7365-3924

\section{Research Article}

Keywords: Conditional Generative Adversarial Networks(CGAN), Covid-19, Time series, Forecasting, Deep Learning

Posted Date: December 13th, 2021

DOI: https://doi.org/10.21203/rs.3.rs-1148944/v1

License: (9) This work is licensed under a Creative Commons Attribution 4.0 International License. Read Full License 


\title{
Time-Series prediction for the epidemic trends of COVID-19 using Conditional Generative adversarial Networks Regression on country-wise case studies
}

\author{
Arnabi Bej $j^{\mathrm{a}} \cdot$ Anasua Sarkar $^{\mathrm{b} *} \cdot \mathrm{Ujjjwal} \mathrm{Maulik}^{\mathrm{c}}$
}

the date of receipt and acceptance should be inserted later

\begin{abstract}
Probabilistic Regression is a statistical technique and a crucial problem in the machine learning domain which employs a set of machine learning methods to forecast a continuous target variable based on the value of one or multiple predictor variables. COVID-19 is a virulent virus that has brought the whole world to a standstill. The potential of the virus to cause inter human transmission makes the world a dangerous place. This thesis predicts the upcoming circumstances of the Corona virus to subside its action. We have performed Conditional GAN regression to anticipate the subsequent Covid-19 cases of 5 countries. The GAN variant CGAN is used to design the model and predict the Covid-19 cases for three months ahead with least error for the dataset provided. Each country is examined individually, due to their variation in population size, tradition, medical management, preventive measures. The analysis is based on confirmed data, as provided by the World Health Organization. This paper investigates how conditional Generative Adversarial Networks (GANs) can be used to accurately exhibit intricate conditional distributions. GANs have got spectacular achievement in producing convoluted highdimensional data, but work done on their use for regression prob- lems is minimal. This paper exhibits how conditional GANs can be employed in probabilistic regression. It is shown that conditional GANs can be used to evaluate a wide range of various distributions and be competitive with existing probabilistic regression models.
\end{abstract}

Keywords Conditional Generative Adversarial Networks(CGAN), Covid- 19,Time series, Forecasting, Deep Learning

${ }^{a}$ A. Bej is associated with the Department of Computer Science and Engineering, Jadavpur University, Kolkata, India, e-mail: arnabib057@gmail.com

$*^{\mathrm{b}}$ A. Sarkar is associated with the Department of Computer Science and Engineering, Jadavpur University, Kolkata, India, e-mail: ashru2006@hotmail.com, anasua.sarkar@jadavpuruniversity.in

${ }^{\mathrm{c}} \mathrm{U}$. Maulik is associated with the Department of Computer Science and Engineering, Jadavpur University, Kolkata, India, e-mail: ujjwal maulik@yahoo.com, ujjwal.maulik@jadavpuruniversity.in 


\section{Introduction}

Since genesis of 2020, COVID-19 has brought entire world to a standstill, infecting greater than 135 million civilians and 2.92M deaths. [1]

This paper gives forecasts for escalation of the COVID19 virus for the widely infected countries like India, USA, Germany, Italy and Spain. Each country is individually inspected, because of their variations in population, habits, customs, health facilities, precautionary measures, etc. The analysis is dependent on actual data, as given by World Health Organization. The deployed deep learning model provides forecasts for new cases and death cases of the population for each country. COVID-19 has a high R0 value (denoting viral infectivity)[2] [3] of 3.25-3.4, represented by wide inter-human spread through the air, which means the flareup is uncontrollable. The main route of COVID-19 transmission is air- borne, thus curbing the source of infection, breaking off the infection route and prevention of endangered masses are the primary ways to discontinue the transmission of COVID-19. Because of this, many countries have taken measures like social distancing, night curfews, shutting down educational institutions, ceasing travel systems to prohibit more transmission of the epidemic [4][5].Due to collected effort of authorities, the public, steps have given rise decreased spread in multiple countries, also the epidemic is decreasing to a large extent. Taking into account the seriousness of the pandemic depicting when the epidemic will terminate is extremely essential for the smooth running of systems of affected countries. Since COVID-19 is basically data on time series so forecasting of future data is extremely needed. Machine Learning and Deep Learning techniques are really beneficial and commonly used in time series prediction. Figure 1 shows transmission and mitigation of Covid19.

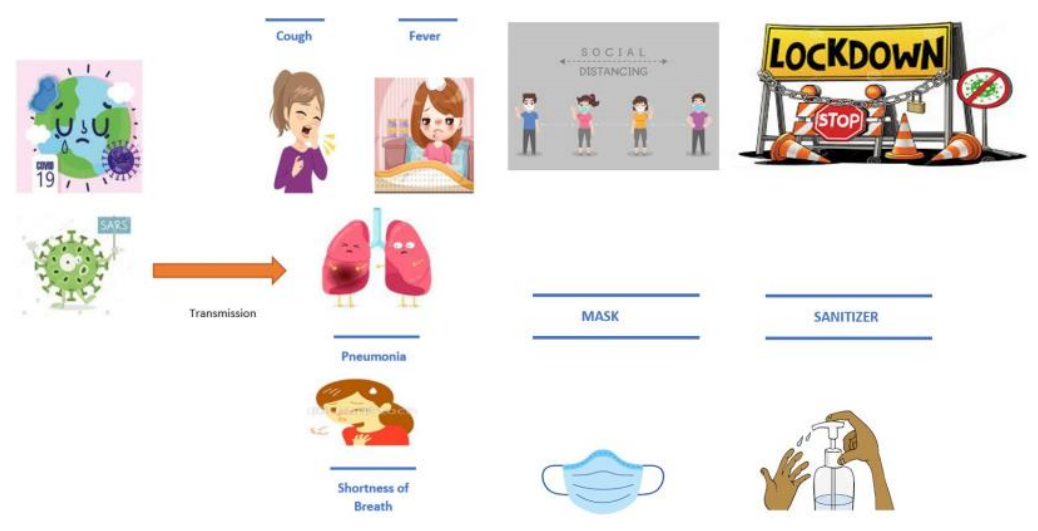

Fig. 1 Transmission and mitigation of COVID 19 


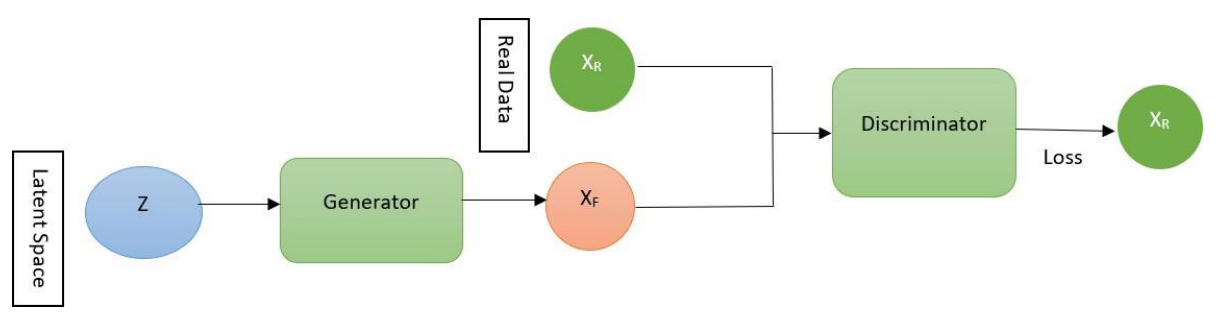

Fig. 2 GAN Architecture

\section{Related Work}

From early centuries the pandemics were prevalent before happening in year 2020. The pace of the entire globe has been stopped due to it. The SARS-COVID-19 virus is originated from Wuhan city, China in the year 2019 [6]. The first death was seen in the month of January that convinced that the virus spread through inter human transmission or very near contact with the person who is suffering from the disease [7]. The United States, Turkey, Russia, the UK and Germany - were the countries mostly affected in December. 46 different countries also saw extreme cases in December. Among them maximum deaths were seen by 31 countries. After fighting long vaccines are available in most countries. Arora et.al. [8] shown the prediction of Covid-19 using LSTM for all states of India with error of 3\%. An ensemble model was designed by Abdulmajeed et al. [9] that took realistic COVID-19 data from Nigeria Center of disease control. Clément Massonnaud et. al [10] Studied the epidemic in France, and also evaluated the effect on healthcare resources for each area of France metropolitan. They produced a SEIR model based on attractive area of the hospitals. Cleo Anastassopoulou et. al. [11] provide estimates of the main epidemiological parameters.

Current Machine learning research is based on deploying models that produces distributions similar to underlying data. Most generative models presume a particular variant. CGAN [13] have exhibited a higher capability to produce authentic samples. Kernel methods, deep neural networks form the non-linear function. Intricate noise forms such as heteroscedastic noise have to be produced using specialized models [12]. Nowadays, research is with image data with a huge organized output space. The categories of various issues like regression is given minimal attention. This paper gives a distribution. Conditional Generative adversarial networks [13] regression is used here.

\section{Methodology}

GAN is a model which produces actual data and has two neural networks, genera- tor and discriminator. Generator produces fake samples. The discriminator has to distinguish whether the input it is getting is G versus real data set. The discrim- inator will output a logic 1 if it finds that its input is more realistic. [14]. GANs 


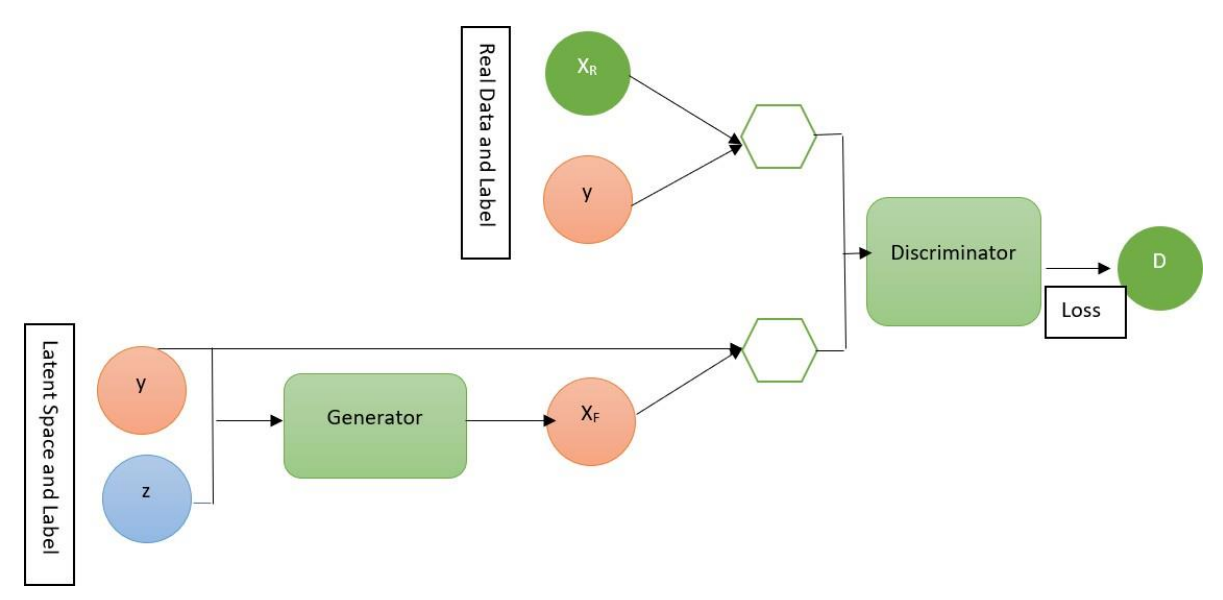

Fig. 3 CGAN Architecture

are continuously evolving domain that provides generative models which produces real like data across different areas of problems for example in image translation tasks like translating pictures of young face to aging face etc. The generator pro- duces a group of samples. These samples alongwith real like data are given to the discriminator and categorized as fake or real. The discriminator thus gets better at distinguishing original and duplicate samples in the subsequent round. After that, the generator will get updated depending upon how perfectly the generator model fooled the discriminator.

Conditional GAN (CGAN) [13] differs from original GAN in the way that it puts an extra condition to manage the production method of the data by con- catenation of an vector $\mathrm{y}$ to both the generator as well as the discriminator. The latent vector $\mathrm{z}$ and vector is input to generator. The samples and the information are fed to the discriminator. Figures 2 and 3 show GAN and CGAN architectures respectively. The CGAN objective function is shown in Figure 4

$$
\min _{G} \max _{D} V(D, G)=\mathbb{E}_{\boldsymbol{x} \sim p_{\text {data }}(\boldsymbol{x})}[\log D(\boldsymbol{x} \mid \boldsymbol{y})]+\mathbb{E}_{\boldsymbol{z} \sim p_{\boldsymbol{z}}(\boldsymbol{z})}[\log (1-D(G(\boldsymbol{z} \mid \boldsymbol{y})))]
$$

Fig. 4 CGAN objective function

\section{Experimental Setup and Implementation}

In this paper we employ the CGAN [13] where conditioning is performed on the generator and discriminator at the time of training by adding some extra infor- 
mation. This information could be anything for example, class labels. Wasserstein GAN [15], can be used also for minimizing loss function[12]. They optimize another loss function, that could affect the performance. We report Root Mean Square Error (RMSE) metric with the experiment. It is one of the important metrics, inspecting importance of the guess of unpredictability is of huge concern.

\subsection{RMSE}

Given a test dataset, (xi, yi) $\mathrm{i}=1 . . \mathrm{N}$, to find the root mean squared error, we first need to evaluate the residuals. Residuals are the substracted value between the actual values and the predicted values. I denoted them by $y_{i}-y_{i}$ where $y_{i}$ is the observed value for the ith observation and $y_{i}$ is the predicted value. RMSE can have any valutes both positive as well as non positive as the depicted value lesser or higher rates the actual value. After performing the residuals square, the squares are averged, and the square root is taken giving us the RMSE. After that the RMSE is deployed as a quantity of the spread of the y values around the depicted y value.

\subsection{Dataset}

We have used the real world WHO - COVID19 dataset taken from// https :

//covid19.who.int/table The dataset consists of new and cumulative cases, new and cumulative deaths which are to be forecasted. The data used for training the model is from 1st January 2020 to 6th April 2021. We have tested the model from 7th April 2021 to 31st July 2021.

\subsection{Preprocessing}

On real world COVID 19 data, training set was preprocessed using minmax scaler preprocessing.

\subsection{Dataset statistics}

After dividing our dataset to training, test groups, we find $80 \%$ of dataset to be training and $20 \%$ of the dataset as test data.

\subsection{Model Architecture}

We have used DNN for CGAN. A six layered network is used for the generator part. The three-layered multi-layer Perceptron gets as input $\mathrm{x}$ and noise $\mathrm{z}$. On the other hand the output illustrations are chained to a three-layered network. Linear activation is used for last layer while Rectified linear unit (RELU) [16] as activation function for remaining layers. RELU performed much better activations such as Leaky-RELU or selu. A four-layered network is used for discriminator. The single 
non-linear layer takes as input $\mathrm{x}$ and $\mathrm{y}$. After that, a three layered multi layered perceptron that employs sigmoid activation function in the final layer takes the concatenated output. The optimizer used is Adam and learning rates used are of values $10^{-2}, 10^{-3}, 10^{-4}$. The number of epochs used was 500. The discriminator and generator training steps ratio was set to 1 .Our model used a batch size of 100 . Noise $\mathrm{z}$ dimension was taken as 1 . the input dimension was 3. Test was performed on a computer having 16GB RAM. CGAN captures very intricate conditional distributions, while different regression methods require various changes.

\subsection{Heteroscedastic noise}

Covid19 real world dataset shows more complex noise. This dataset employs heteroscedastic noise. CGAN generates more realistic samples and captures the heteroscedastic noise structure very well. CGAN can learn to model any type of noise very easily.

\subsection{Increasing the dimensionality of noise}

Employing a larger dimensional noise can assist get enhanced behaviour for problems with complex noise.

The proposed framework of the model is shown in Figure 5. The generator network structure and discriminator network structure are shown in Figure 6 and 7 respectively.

\subsection{Mechanism of Rolling Update}

In COVID-19 cases for achieving extended forecasts, a rolling update process is employed. It is used to upgrade the training example sequence depending on the present forecast values instantaneously for the purpose of training the model. It is actually done to enhance the rolling control of feedback evaluation in a bounded amount of time.

There are different parameters in the model. The input time step is taken as 3, which implies that new cases count of the next day is dependent on the cases of the last three days. The count of hidden neurons is 40 , This is the input which is fed to hidden layer. The model used Adam optimizer, training of 500epochs.

\section{Results and Discussions}

Results of COVID cases and deaths of five countries India, Italy, Spain, USA, Germany are visualised graphically and RMSE classification metric calculation. Covid-19 new cases and death cases predicted values of the five countries are visualised from figures 8-17 The blue curve represents the training portion. The green curve represents true data from 7 th April 2021 to 31st July,2021. And the yellow curve shows the predicted values. 


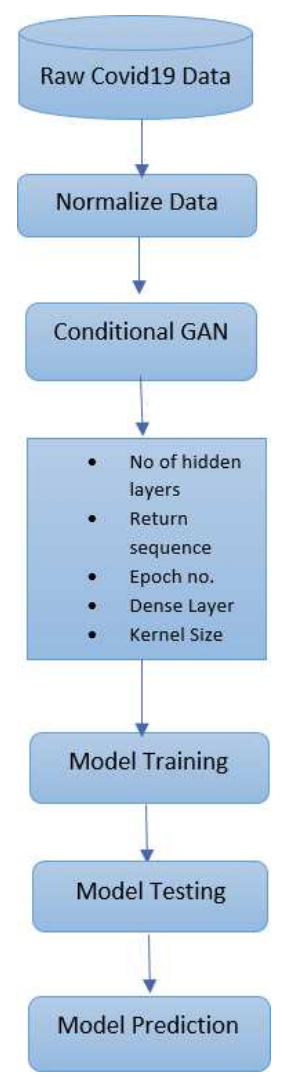

Fig. 5 Proposed Methodology Framework

\section{Covid-19 Forecasting of daily new cases}

The COVID19 true and predicted data of the countries India,USA, Spain, Italy, Germany are visualised graphically in the figures $8,10,12,14,16$. The blue coloured curve depicts actual values and red coloured curve depicts forecasted values.

\section{Covid-19 Forecasting of daily new death cases}

The COVID19 true and predicted data of the countries India,USA, Spain, Italy, Germany are visualised graphically in the figures $9,11,13,15,17$. The blue coloured curve depicts actual values and red coloured curve depicts forecasted values.

The analysis of the graphs and classification metric implies that graphs and CGAN results are very close to the real cases of the five countries. The alpha value 


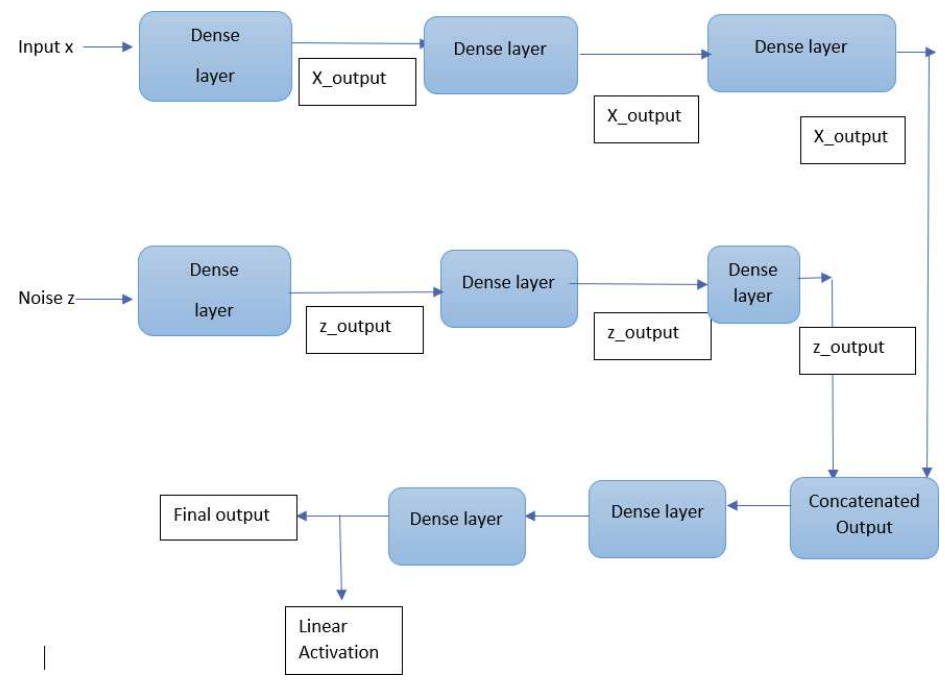

Fig. 6 Generator network structure

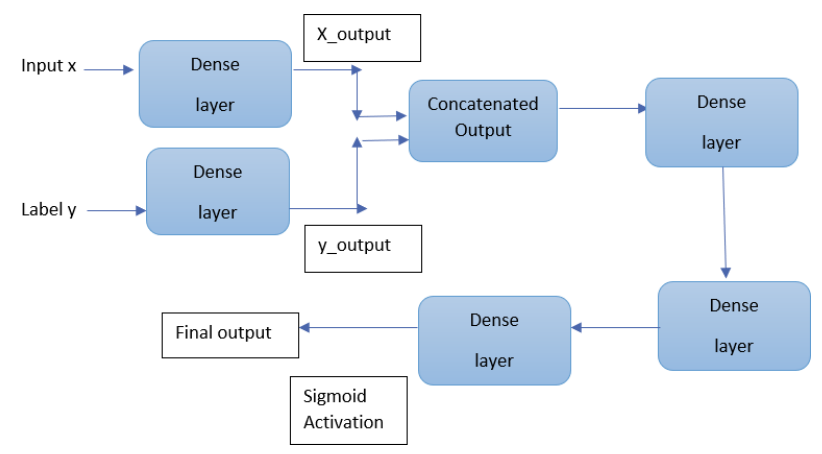

Fig. 7 Discriminator network structure

or significance level is considered to be 0.05 or $5 \%$. When P-value is smaller than alpha value, the results are statistically significant.

Alpha value is actually a threshold p-value, that is actually decided by a team who conducted the test. It is decided before performing significance test.(Z Test) To find the reported and forecasted value difference, Root Mean Square Error is to be found, by the equation: RMSE $=\frac{\sum_{1}^{n}(y,-\hat{y}) i^{2}}{n}$ Root Mean Squared Error values and P-values are shown in Table 2 and Table 3

Mean absolute percentage error (MAPE) It evaluates how good a prediction model is. It calculates this accuracy in terms of percentage. 


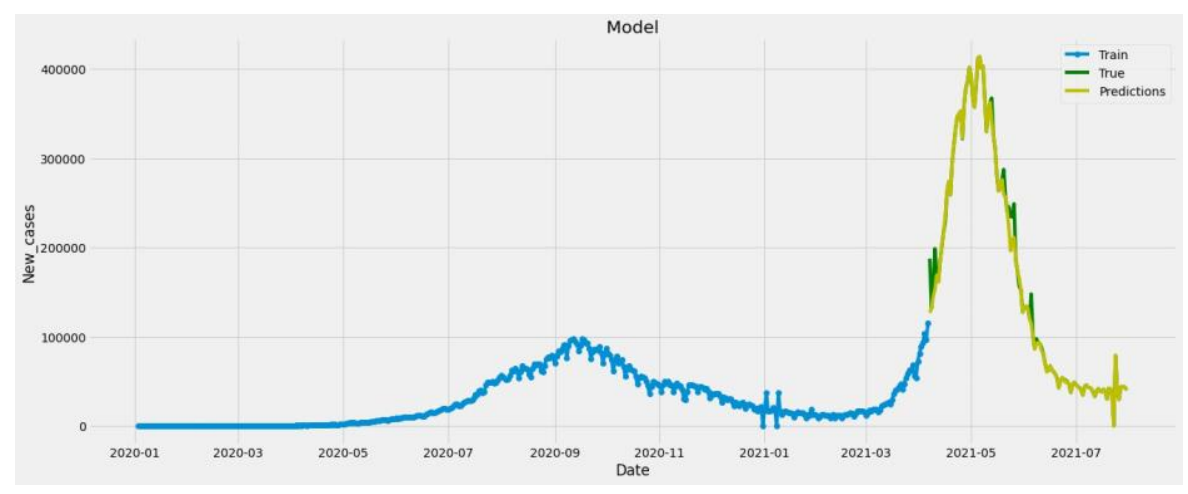

Fig. 8 India New Cases

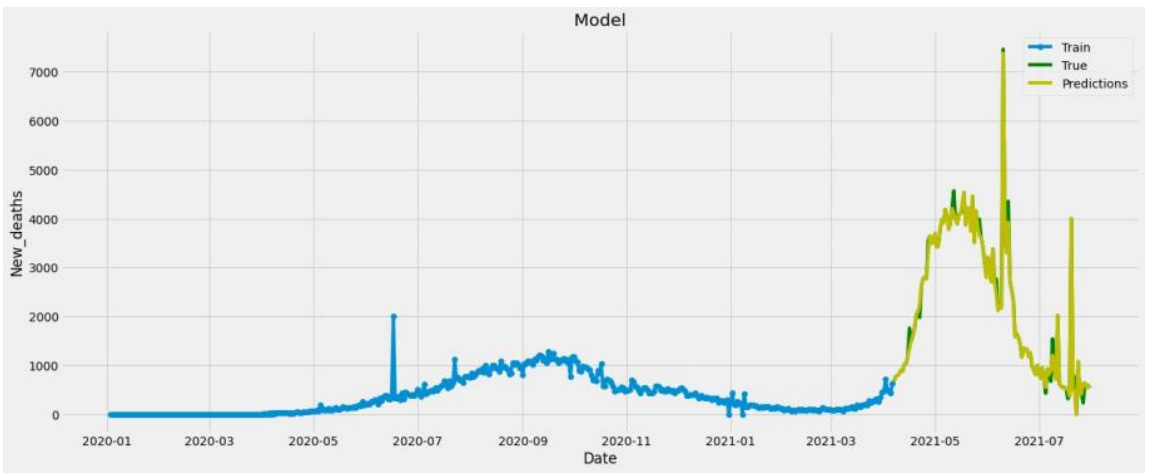

Fig. 9 India New Deaths

MAPE output is non-negative floating point. The best value is 0.0 . But note the fact that bad predictions can lead to arbitarily large MAPE values, especially if some $y_{\text {true }}$ values are very close to zero. Note that we return a large value instead of inf when $y_{\text {true }}$ is zero. MAPE is calculated by the equation MAPE $=$

$\Sigma$

Mean absolute percentage error (MAPE) is shown in Table 4 It gives minimum errors in our predicted data.

R2 Score R-squared (R2) or Coefficient of Determination is a statistical mea- sure that represents the proportion of the variance for a dependent variable that's explained by an independent variable or variables in a regression model. It is also

called the coefficient of determination. $R^{2} \quad$ Score $=1-\quad \begin{array}{r}\left(y_{i}-y_{i}\right)^{2} \\ \left(y_{i}-y^{2}\right.\end{array}$

Coefficient of Determination is shown in table 5

Explained variance score It is used to measure the discrepancy between a model and actual data. In other words, it's the part of the model's total variance that is explained by factors that are actually present and isn't due to error variance. The great score is 1.0 and lesser values are bad. 


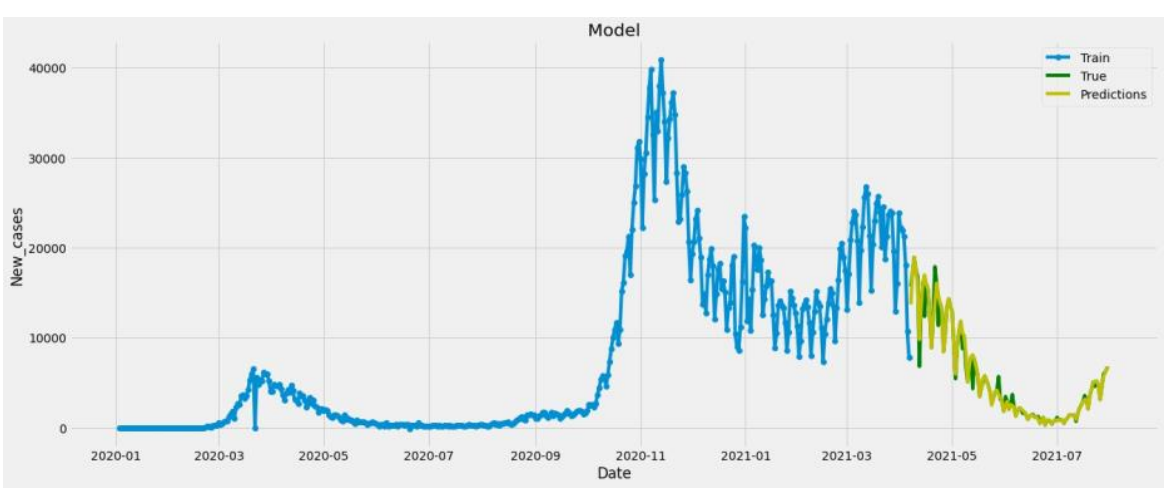

Fig. 10 Italy New Cases

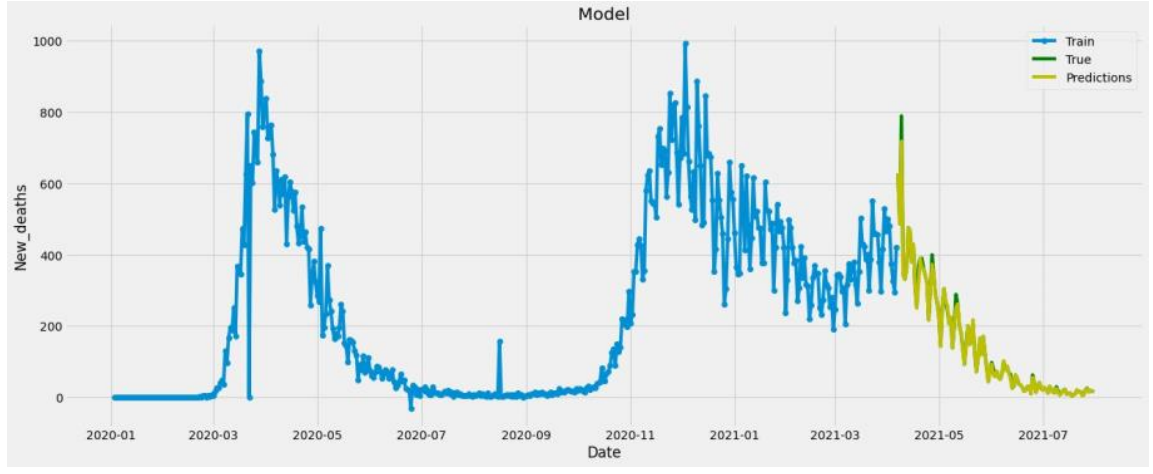

Fig. 11 Italy New Deaths

Explained variance is shown in figure 6.

Table 7 shows predicted and reported data of the new COVID cases of India, Italy, Germany, Spain, USA from 6th June 2021 to 10th June, 2021. The values show that reported and predicted data are very close to each other.

Table 1 Choosing Data used for experiment

\begin{tabular}{lll}
\hline Country & Confirmed cases & Death cases \\
\hline India & $1.1 .2020-06.04 .2021$ & $1.1 .2020-06.04 .2021$ \\
Germany & $1.1 .2020-06.04 .2021$ & $1.1 .2020-06.04 .2021$ \\
USA & $1.1 .2020-06.04 .2021$ & $1.1 .2020-06.04 .2021$ \\
Italy & $1.1 .2020-06.04 .2021$ & $1.1 .2020-06.04 .2021$ \\
Spain & $1.1 .2020-06.04 .2021$ & $1.1 .2020-06.04 .2021$ \\
\hline
\end{tabular}




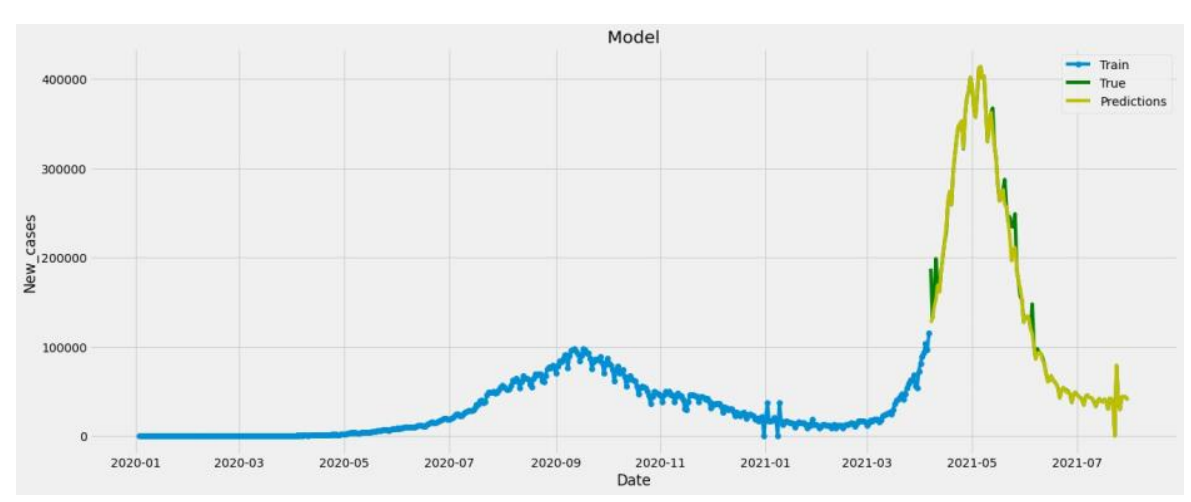

Fig. 12 Germany New Cases

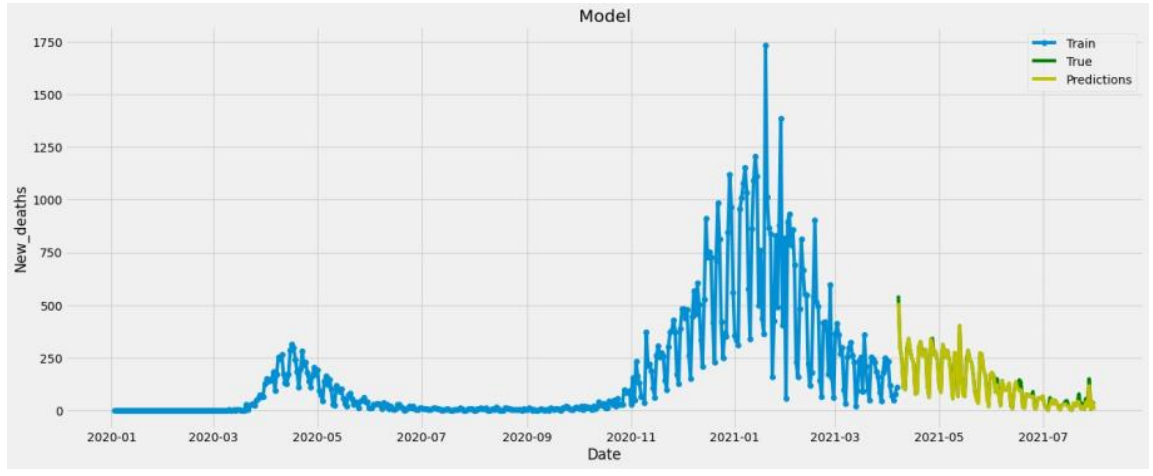

Fig. 13 Germany New Deaths

Table 2 RMSE Values

\begin{tabular}{lllll}
\hline Country & New Cases & Cumulative Cases & New Deaths & Cumulative deaths \\
\hline India & 557.4 & 448.49 & 163.35 & 503.44 \\
Germany & 665.65 & 231.3 & 412.6 & 242.06 \\
USA & 709.35 & 542.8 & 587.54 & 142.43 \\
Italy & 734.7 & 681.7 & 308.5 & 221.4 \\
Spain & 603.56 & 126.3 & 253.8 & 203.4 \\
\hline
\end{tabular}

\section{Discussions and Suggestions}

\subsection{Development of Vaccine and its side effects}

Presently, there are 115 candidate vaccines for [17] COVID-19. In total there are 33 candidate vaccines in stage 3 clinical trials. So far, 15 vaccines have been authorized across several countries. After a thorough evaluation of phase 3 clinical trial data major side effects after getting the Sputnik V vaccine were flu-like illness, headache, fatigue, and reactions at the injection site. It is based on surveys from 12,296 


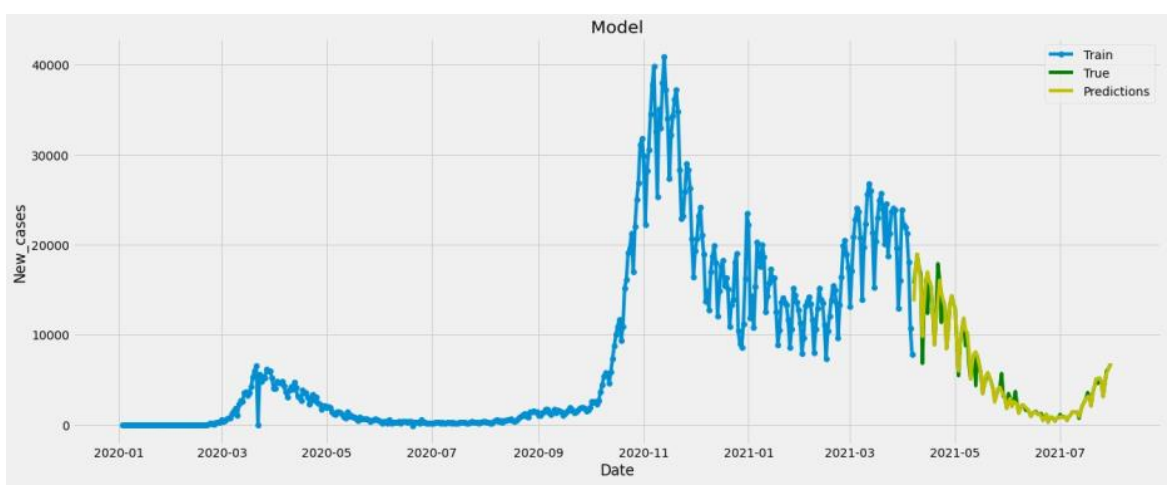

Fig. 14 USA New Cases

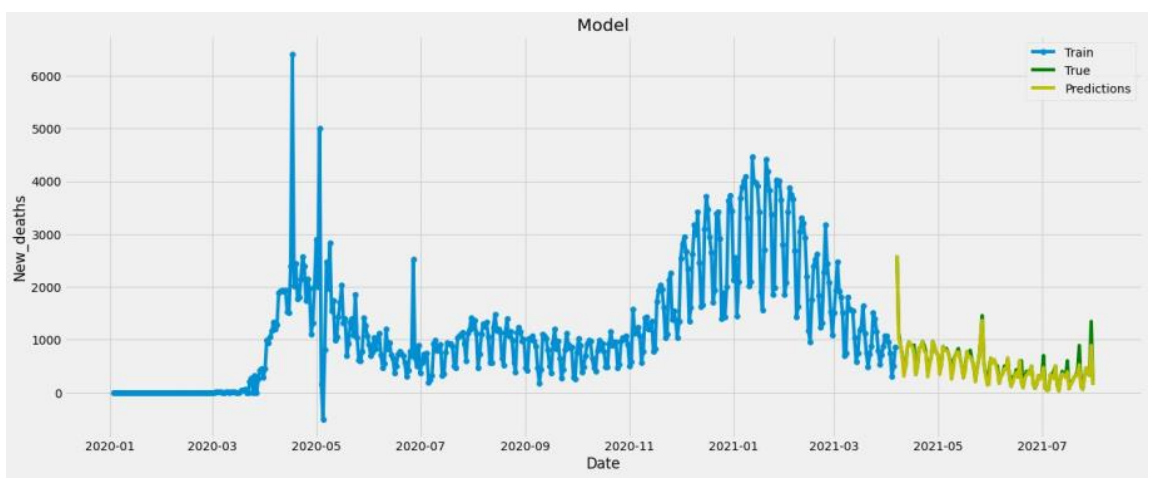

Fig. 15 USA New Deaths

Table 3 P values

\begin{tabular}{lllll}
\hline Country & New Cases & Cumulative Cases & New Deaths & Cumulative deaths \\
\hline India & 0.02 & 0.007 & $0.008 e^{-2}$ & $2.15 e^{-1}$ \\
Germany & 0.003 & $2.43 e^{-1}$ & 0.0016 & $3.3 e^{-2}$ \\
USA & $3.6 e^{-2}$ & $2.6 e^{-1}$ & $2.87 e^{-1}$ & $3.05 e^{-2}$ \\
Italy & $2.18 e^{-2}$ & $1.28 e^{-1}$ & $3.41 e^{-1}$ & $2.11 e^{-2}$ \\
Spain & 0.014 & $2.6 e^{-2}$ & $3.2 e^{-1}$ & $2.5 e^{-2}$ \\
\hline
\end{tabular}

participants who had received two doses of either the vaccine or the placebo. There is quite a lot of turmoil regarding the vaccine, with some scientists unsure of the data and remaining are scared of side effects that the shot employs[18] However, 64 countries have been using the vaccine, in addition to Russia. 


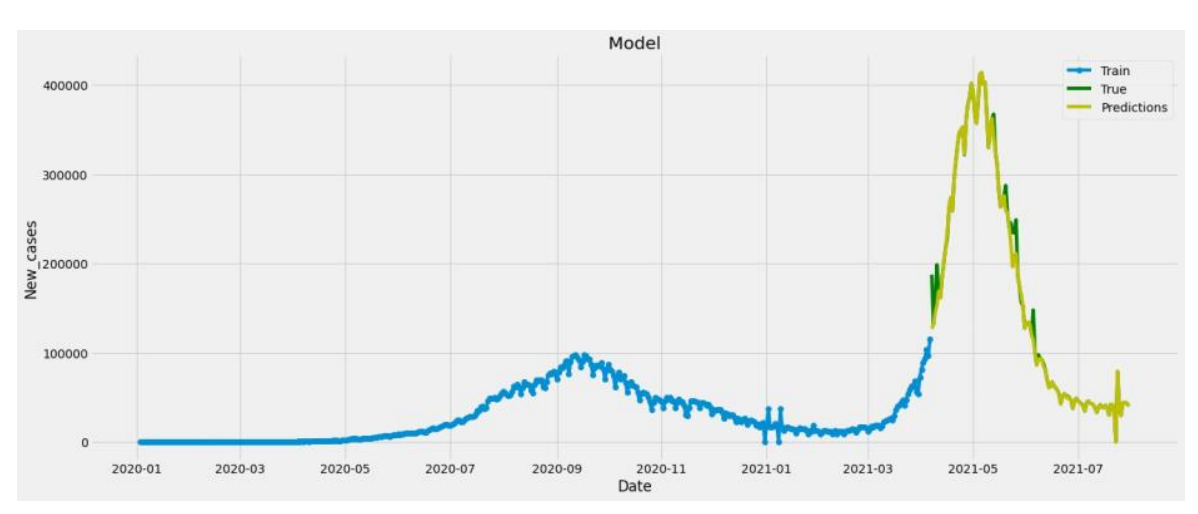

Fig. 16 Spain New Cases

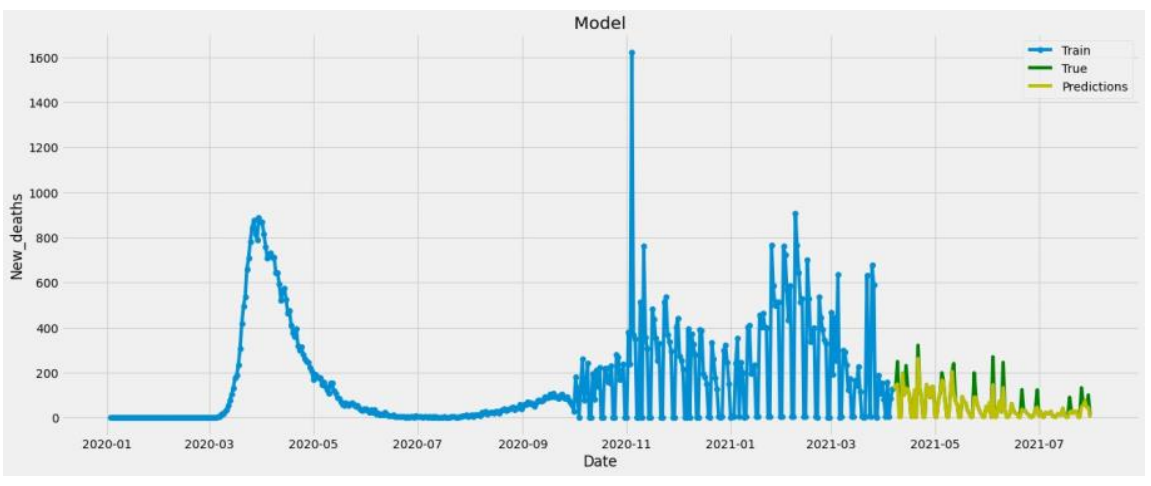

Fig. 17 Spain New Deaths

Table 4 mean absolute percentage error

\begin{tabular}{lllll}
\hline Country & New Cases & Cumulative Cases & New Deaths & Cumulative deaths \\
\hline India & 2.38 & 2.49 & 1.26 & 0.66 \\
Germany & 0.54 & 2.40 & 0.84 & 3.42 \\
USA & 0.84 & 2.64 & 0.40 & 3.52 \\
Italy & 0.59 & 2.18 & 3.33 & 2.38 \\
Spain & 1.94 & 3.87 & 3.04 & 1.91 \\
\hline
\end{tabular}

\subsection{Transmission of Covid19 through air}

Covid-19 is a disease that spreads through air and is a danger to the world, according to various researchers. Aerosol transmission is another mode of transmission of Covid-19. Not going outside house, frequent cleaning of hands and maintaining social distance are compulsory measures for avoiding airborne transferral of COVID-19 according to WHO with various other international authorities. Wearing face masks is extremely essential to stop inhaling aerosol. Social gathering must be avoided. aerosol transmission is not universally accepted till now but some re- 
Table $5 \mathrm{R}^{2}$ score, the coefficient of determination

\begin{tabular}{lllll}
\hline Country & New Cases & Cumulative Cases & New Deaths & Cumulative deaths \\
\hline India & 0.87 & 0.77 & 0.89 & 0.84 \\
Germany & 0.768 & 0.8 & 0.9 & 0.96 \\
USA & 0.75 & 0.86 & 0.78 & 0.98 \\
Italy & 0.83 & 0.75 & 0.93 & 0.88 \\
Spain & 0.89 & 0.94 & 0.84 & 0.95 \\
\hline
\end{tabular}

Table 6 explained variance score

\begin{tabular}{lllll}
\hline Country & New Cases & Cumulative Cases & New Deaths & Cumulative deaths \\
\hline India & 0.54 & 0.60 & 0.61 & 0.62 \\
Germany & 0.68 & 0.84 & 0.56 & 0.64 \\
USA & 0.51 & 0.90 & 0.76 & 0.50 \\
Italy & 0.73 & 0.51 & 0.633 & 0.60 \\
Spain & 0.63 & 0.85 & 0.71 & 0.85 \\
\hline
\end{tabular}

searchers found evidences of it [19]. So, precautionary measures must be taken to be prevented from the dangerous effects of the pandemic.

\section{Conclusion}

Covid-19 has [20] brought the world to a standstill due to its rampant spread. World has to cope with the with the pandemic as it is not known how long the pandemic will be there. We used deep learning model to predict new cases and new death cases for India, USA, Spain, Italy and Germany of COVID19 in this paper. Collecting the data of Covid-19 really hard because of its limited availability for forecasting time series data. Conditional Generative adversarial network is used to model the predictions. The Root mean squared error is evaluated here. The predictions of this paper's CGAN model which provides current situation's results is close to actual Covid-19 data. This paper is the first evaluation of India, USA, Spain, Italy, Germany Covid-19 new cases and deaths. Our study will be essential for the five countries to take proper steps before being dominated by Covid-19 pandemic. We can inspect the losses incurred by the economies of the countries at the end of Covid-19 and take precautionary steps to overcome it, which can help the countries to come back into normal conditions. The Covid-19 new cases and death cases of several other countries can be predicted lateron.

\section{Declaration of Competing Interest}

The authors declare that they have no familiar monetary motives that could have portrayed to impact the task presented in this paper. 
Table 7 True and depicted daily new cases comparison

\begin{tabular}{|c|c|c|c|}
\hline Country & Date & Reported data & Predicted data \\
\hline India & $\begin{array}{l}06.6 .2021 \\
07.6 .2021 \\
08.6 .2021 \\
09.6 .2021 \\
10.6 .2021 \\
\end{array}$ & $\begin{array}{l}100636 \\
86498 \\
92596 \\
93463 \\
92291\end{array}$ & $\begin{array}{l}100321 \\
86212 \\
92321 \\
93112 \\
92345\end{array}$ \\
\hline Germany & $\begin{array}{l}06.6 .2021 \\
07.6 .2021 \\
08.6 .2021 \\
09.6 .2021 \\
10.6 .2021 \\
\end{array}$ & $\begin{array}{l}1964 \\
1444 \\
2253 \\
3275 \\
2747 \\
\end{array}$ & $\begin{array}{l}1912 \\
1421 \\
2289 \\
3264 \\
2732 \\
\end{array}$ \\
\hline USA & $\begin{array}{l}06.6 .2021 \\
07.6 .2021 \\
08.6 .2021 \\
09.6 .2021 \\
10.6 .2021 \\
\end{array}$ & $\begin{array}{l}5395 \\
15496 \\
13013 \\
18647 \\
14545 \\
\end{array}$ & $\begin{array}{l}5356 \\
15400 \\
13000 \\
18670 \\
14500 \\
\end{array}$ \\
\hline Italy & $\begin{array}{l}06.6 .2021 \\
07.6 .2021 \\
08.6 .2021 \\
09.6 .2021 \\
10.6 .2021 \\
\end{array}$ & $\begin{array}{l}2275 \\
1270 \\
1894 \\
2198 \\
2078 \\
\end{array}$ & $\begin{array}{l}2289 \\
1265 \\
1800 \\
2450 \\
2043 \\
\end{array}$ \\
\hline Spain & $\begin{array}{l}06.6 .2021 \\
07.6 .2021 \\
08.6 .2021 \\
09.6 .2021 \\
10.6 .2021\end{array}$ & $\begin{array}{l}3 \\
9542 \\
3504 \\
4427 \\
14004\end{array}$ & $\begin{array}{l}10 \\
9456 \\
3605 \\
4489 \\
14390\end{array}$ \\
\hline
\end{tabular}

\section{References}

1. Kathryn H Jacobsen. Will covid-19 generate global preparedness? The Lancet, 395(10229):10131014, 2020.

2. Qun Li, Xuhua Guan, Peng Wu, Xiaoye Wang, Lei Zhou, Yeqing Tong, Ruiqi Ren, Kathy SM Leung, Eric HY Lau, Jessica Y Wong, et al. Early transmission dynamics in wuhan, china, of novel coronavirus-infected pneumonia. New England journal of medicine, 2020.

3. Nian Shao, Jin Cheng, and Wenbin Chen. The reproductive number r0 of covid-19 based on estimate of a statistical time delay dynamical system. MedRxiv, 2020.

4. Moritz UG Kraemer, Chia-Hung Yang, Bernardo Gutierrez, Chieh-Hsi Wu, Brennan Klein, David M Pigott, Louis Du Plessis, Nuno R Faria, Ruoran Li, William P Hanage, et al. The effect of human mobility and control measures on the covid-19 epidemic in china. Science, 368(6490):493-497, 2020.

5. Mehmet Ozaslan, Muhamad Safdar, I Halil Kilic, and Rozhgar A Khailany. Practical measures to prevent covid-19: a mini-review. J Biol Sci, 20:100-102, 2020.

6. Chaolin Huang, Yeming Wang, Xingwang Li, Lili Ren, Jianping Zhao, Yi Hu, Li Zhang, Guohui Fan, Jiuyang Xu, Xiaoying Gu, et al. Clinical features of patients infected with 2019 novel coronavirus in wuhan, china. The lancet, 395(10223):497-506, 2020.

7. Chen Wang, Peter W Horby, Frederick G Hayden, and George F Gao. A novel coronavirus outbreak of global health concern. The lancet, 395(10223):470-473, 2020.

8. Parul Arora, Himanshu Kumar, and Bijaya Ketan Panigrahi. Prediction and analysis of covid-19 positive cases using deep learning models: A descriptive case study of india. Chaos, Solitons \& Fractals, 139:110017, 2020.

9. Kabir Abdulmajeed, Monsuru Adeleke, and Labode Popoola. Online forecasting of covid- 19 cases in nigeria using limited data. Data in brief, 30:105683, 2020.

10. Clément Massonnaud, Jonathan Roux, and Pascal Crépey. Covid-19: Forecasting short term hospital needs in france. medrxiv, 2020.

11. Cleo Anastassopoulou, Lucia Russo, Athanasios Tsakris, and Constantinos Siettos. Data-based analysis, modelling and forecasting of the covid-19 outbreak. PloS one, 15(3):e0230405, 2020.

12. Quoc V Le, Alex J Smola, and Stéphane Canu. Heteroscedastic gaussian process regression. In Proceedings of the 22nd international conference on Machine learning, pages 489-496, 2005.

13. Mehdi Mirza and Simon Osindero. Conditional generative adversarial nets. arXiv preprint arXiv:1411.1784, 2014.

14. Xudong Mao, Qing Li, Haoran Xie, Raymond YK Lau, Zhen Wang, and Stephen Paul Smolley. Least squares generative adversarial networks. In Proceedings of the IEEE international conference on computer vision, pages 2794-2802, 2017.

15. Martin Arjovsky, Soumith Chintala, and Léon Bottou. Wasserstein generative adversarial networks. In International conference on machine learning, pages 214-223. PMLR, 2017.

16. Djork-Arné Clevert, Thomas Unterthiner, and Sepp Hochreiter. Fast and accurate deep network learning by exponential linear units (elus). arXiv preprint arXiv:1511.07289, 2015.

17. Guido Forni and Alberto Mantovani. Covid-19 vaccines: where we stand and challenges ahead. Cell Death \& Differentiation, 28(2):626-639, 2021.

18. Victoria Male. Are covid-19 vaccines safe in pregnancy? Nature Reviews Immunology, pages 1-2, 2021.

19. L Morawska and DK Milton. It is time to address airborne transmission of coronavirus disease 2019 (covid-19) clin. Infect. Dis, 6, 2020. 20 Lee H Schwamm, Alistair Erskine, and Adam Licurse. A digital embrace to blunt the curve of covid19 pandemic. NPJ digital medicine, 3(1):1-3, 2020. 